\title{
NIVEL SÉRICO DE ESTRADIOL A LA MONTA Y SU EFECTO SOBRE LA TASA DE CONCEPCIÓN EN ALPACAS
}

\author{
Serum Oestradiol Level During Mating And Its Effect on Conception \\ Rate IN THE AlPaCA
}

\author{
Arturo Díaz C. ${ }^{1}$, Héctor Huamán U. ${ }^{2, u ̈, ~ J o s e ́ ~ C a m a c h o ~ S . ~}{ }^{3}$, \\ Antonio Ampuero B. ${ }^{3}$, Daniel Quispe M. ${ }^{4}$, Wilfredo Huanca L.,
}

\section{Resumen}

\begin{abstract}
El objetivo del estudio fue determinar el nivel sérico de estradiol en alpacas adultas al momento del primer servicio posparto y su efecto sobre la tasa de concepción. Se seleccionó 85 alpacas adultas, vacías y sin cría, y se sirvieron con machos de fertilidad comprobada, previa evaluación de conducta de receptividad frente al macho. Luego de la monta, se colectó una muestra de sangre y el nivel de estradiol en el suero se analizó por radioinmunoensayo. La concepción se determinó por ecografía a los 25-27 días poscópula. Los valores de estradiol se dividieron en dos grupos: A $(<0.5 \mathrm{pg} / \mathrm{mL})$ y B $(>0.5 \mathrm{pg} / \mathrm{mL})$, donde la media fue de $0.78 \pm 0.39 \mathrm{pg} / \mathrm{mL}$. La tasa de concepción en alpacas con bajo nivel de estradiol en el momento de la monta fue de $52.5 \%$ y en alpacas con alto nivel de estradiol (>0.5 pg/mL) fue de $66.7 \%$, sin diferencias estadísticas; indicando la ausencia de una influencia de los niveles de estradiol sobre la tasa de concepción.
\end{abstract}

Palabras clave: alpaca, estradiol, concepción, radioinmunoensayo

\section{Abstract}

The aim of the study was to determine the serum oestradiol level in adult alpacas during the first post-partum mating and its effect on conception rate. It was selected 85 non-lactating female adult alpacas without calf at foot. Sexual receptive females were mated with a male of proven fertility. Blood samples were collected at mating and the level of oestradiol in serum was determined by radioimmunoassay. Conception was determined by ultrasound at 25-27 days of mating. Oestradiol values were distributed in two groups: $\mathrm{A}(<0.5 \mathrm{pg} / \mathrm{mL})$ and $\mathrm{B}(>0.5 \mathrm{pg} / \mathrm{mL})$, where the mean was $0.78 \pm 0.39 \mathrm{pg} / \mathrm{mL}$. Conception rate in alpacas with low oestradiol level at time of mating was $52.5 \%$ and in alpacas with high level was $66.7 \%$ without significant difference. Results indicate the lack of influence of oestradiol levels on conception rate.

Key words: alpaca, oestradiol, conception, radioimmunoassay

\footnotetext{
${ }^{1}$ Laboratorio de Reproducción Animal, ${ }^{2}$ Laboratorio de Bioquímica, Nutrición y Alimentación Animal, ${ }^{3}$ Laboratorio de Zootecnia y Producción Agropecuaria, Facultad de Medicina Veterinaria, Universidad Nacional Mayor de San Marcos, Lima

${ }^{4}$ CIP Quimsachata, EE ILLPA-INIA. Puno

${ }^{5}$ E-mail: whuanca2002@yahoo.com
} 


\section{INTRODUCCIÓN}

Los camélidos se incluyen en el grupo de "ovuladores inducidos", donde la cópula es necesaria para producir el estímulo para la producción y liberación de las hormonas hipofisiarias requeridas para la ovulación (Novoa y Leyva, 1996). Las características conductuales con periodos largos de aceptación al macho y periodos muy cortos de rechazo, reflejan las ondas de crecimiento, maduración y atresia folicular y la presencia continua de folículos que secretan cantidades adecuadas de estrógeno, hormona responsable de la manifestación de receptividad (Fernández Baca, 1971).

Las alpacas presentan una alta tasa de mortalidad embrionaria, que puede llegar al $50 \%$ dentro de los primeros 35 días de gestación (Fernández-Baca et al., 1970). Asimismo, estudios realizados por Leyva y García (1999) reportan $12 \%$ de mortalidad embrionaria en los primeros 5 días post-ovulación, mortalidad que estaría causada por un efecto negativo del estradiol sobre el desarrollo del cuerpo lúteo en ese periodo (Leyva y García, 2000). Por otro lado, Sumar et al. (1988) sugieren que el estradiol tiene un rol importante sobre la ovulación, debido a una elevación brusca de 100-200 a 700 pmol después de la cópula, pero antes de la ovulación.

Existen contradicciones sobre las concentraciones de estradiol y actividad folicular. Bravo et al. (1990) sugieren que las concentraciones de estradiol están positivamente asociadas con la actividad folicular. Estos autores reportan que las concentraciones de sulfato de estrona en la orina estuvieron elevadas en el día del servicio (30.2 ng/mg), indicando la presencia de folículos antrales grandes, para disminuir a las 24 y 48 horas post-servicio (Bravo et al., 1992). Por otro lado, Sumar (1997) sugiere que no existe relación directa entre función ovárica y receptividad sexual en alpacas, porque muchas de ellas muestran receptividad, aun sin presentar folículos o con folículos menores de $3 \mathrm{~mm}$.

La relación entre la conducta de receptividad de alpacas hembras con la secreción de hormonas esteroides ováricas y su efecto sobre la tasa de concepción, no ha sido claramente establecida; por lo que el presente estudio, tuvo como objetivo determinar el posible efecto de los niveles séricos de estradiol sobre la tasa de concepción en la alpaca.

\section{Materiales y Métodos}

\section{Lugar de Estudio y Animales}

El trabajo se realizó entre enero y abril de 2009, en el Centro de Investigación y Producción (CIP) Quimsachata, anexo de la Estación Experimental ILLPA, Instituto Nacional de Investigación y Extensión Agraria (INIA) - Puno, Perú. El Centro está ubicado en la provincia de Lampa, a $4200 \mathrm{msnm}$, correspondiendo a una zona agroecológica de puna seca, piso altitudinal subalpino, cuyo clima es de tipo semi-seco frío. La temperatura media es de $7{ }^{\circ} \mathrm{C}$ (máxima $18^{\circ} \mathrm{C}$ y mínima de $-3{ }^{\circ} \mathrm{C}$ ). La precipitación pluvial anual oscila entre 400 y 688, siendo los meses más húmedos de diciembre a marzo.

Se seleccionaron 85 hembras, vacías, mayores de tres años, sin cría al pie, con historial reproductivo de haber tenido al menos un parto anterior y con un descanso sexual $>20$ días posparto. Todos los animales recibieron las mismas condiciones de manejo y alimentación, bajo pastoreo extensivo con pasturas naturales conformadas mayoritariamente por gramíneas, ciperáceas, juncáceas y leguminosas.

\section{Procedimiento Metodológico}

Las hembras fueron servidas con machos enteros, de fertilidad comprobada, previa evaluación de conducta de receptividad 
Cuadro 1. Tasa de concepción en alpacas con bajos (grupo A) y altos (grupo B) niveles séricos de estradiol en el momento de la monta

\begin{tabular}{cccc}
\hline Variable & $\begin{array}{c}\text { Grupo A } \\
(\mathrm{n}=61)\end{array}$ & $\begin{array}{c}\text { Grupo B } \\
(\mathrm{n}=24)\end{array}$ & $\begin{array}{c}\text { Total }^{3} \\
(\mathrm{n}=85)\end{array}$ \\
\hline \multirow{2}{*}{ Preñada } & $52.5 \%$ & $66.7 \%$ & $56.5 \%$ \\
& $(\mathrm{n}=32)$ & $(\mathrm{n}=16)$ & $(\mathrm{n}=48)$ \\
\multirow{2}{*}{ Vacía } & $47.5 \%$ & $33.3 \%$ & $43.5 \%$ \\
& $(\mathrm{n}=29)$ & $(\mathrm{n}=8)$ & $(\mathrm{n}=37)$ \\
\hline \multirow{2}{*}{ Total } & $100.0 \%$ & $100.0 \%$ & $100.0 \%$ \\
& $(\mathrm{n}=61)$ & $(\mathrm{n}=24)$ & $(\mathrm{n}=85)$ \\
\hline
\end{tabular}

Nivel sérico de estradiol: ${ }^{1} 0.02 \pm 0.02 ;{ }^{2} 2.70 \pm 1.06 ;{ }^{3} 0.78 \pm 0.39 \mathrm{pmol} / \mathrm{mL}$

Cuadro 2. Efecto de la edad de la madre, tiempo de cópula, e intervalo parto-monta sobre el porcentaje de concepción en relación con el nivel de estradiol a la monta (Grupo A: $<0.5 \mathrm{pg} / \mathrm{mL}$, y Grupo B: $>0.5 \mathrm{pg} / \mathrm{mL}$ )

\begin{tabular}{lllccc}
\hline Variable & & & $\begin{array}{c}\text { Grupo A } \\
(n=61)\end{array}$ & $\begin{array}{c}\text { Grupo B } \\
(n=24)\end{array}$ & $\begin{array}{c}\text { Total } \\
(n=85)\end{array}$ \\
\hline Edad & 3 & $(n=19)$ & 56.3 & 66.7 & $57-9$ \\
$($ años $)$ & $4-6$ & $(n=30)$ & 65.0 & 70.0 & 66.7 \\
& $>6$ & $(n=36)$ & 40.0 & 63.7 & 47.2 \\
Tiempo de & $15-20$ & $(n=33)$ & 60.0 & 33.3 & 50.0 \\
cópula & $>20$ & $(n=39)$ & 48.2 & 55.6 & 54.5 \\
$($ minutos $)$ & No registro $(n=5)$ & 66.7 & $90.0^{\mathrm{b}}$ & 59.0 \\
& $<20$ & $(\mathrm{n}=9)$ & 50.0 & 50.0 & 60.0 \\
Intervalo parto- & $21-30$ & $(n=16)$ & 45.5 & 66.7 & 55.6 \\
monta & $>30$ & $(n=32)$ & 50.0 & 80.0 & 43.8 \\
$($ días $)$ & No registro $(n=28)$ & -- & -- & 59.4 \\
& & & 52.5 & 66.7 & -- \\
\hline Total & & & & 56.5 \\
\hline
\end{tabular}

${ }^{a, b}$ Superíndices diferentes dentro de filas indican diferencia estadística $(p<0.05)$

frente al macho. Se consideró el día de la cópula como el día 0 , y como hembra receptiva la que adoptó la posición decúbito ventral frente a la presencia del macho. Luego de la monta, se les colectó 5-7 ml de sangre por punción de la vena yugular. Las muestras fueron centrifugadas dentro de las dos horas a $3000 \mathrm{rpm}$ por 10 minutos y el suero resultante se almacenó a $-20^{\circ} \mathrm{C}$ hasta su análisis. 
La concepción se determinó por ecografía a los 25-27 días poscópula. El criterio para determinar a un animal gestante fue la observación de una estructura anecoica en el útero correspondiente a la vesícula embrionaria y la presencia del embrión.

El estradiol se determinó mediante la técnica de radioinmunoensayo (RIA) en el Laboratorio de Reproducción Animal de la Facultad de Medicina Veterinaria de la Universidad Nacional Mayor de San Marcos, Lima, utilizando un kit comercial (DPC, California, USA).

Para efectos del análisis de la información, los niveles de estradiol fueron categorizados en dos grupos, en base a la concentración de estradiol en el día de la monta, según el reporte en camellos de Deen et al. (2007): Grupo A (nivel basal de estradiol $<0.5 \mathrm{pg} / \mathrm{mL}$ ) y Grupo B (Nivel de estradiol $>0.5 \mathrm{pg} / \mathrm{mL}$ ). Además, se consideró la variable preñada o vacía. Los datos fueron analizados mediante la prueba de Chi-cuadrado.

\section{Resultados}

Las tasas de concepción en el grupo de bajo $(<0.5 \mathrm{pg} / \mathrm{mL})$ y alto $(>0.5 \mathrm{pg} / \mathrm{mL})$ nivel sérico de estradiol se presentan en el Cuadro 1. Se observa que un mayor número de animales resultó con bajo nivel de estradiol.

La mayor tasa de concepción se presentó en las alpacas de 4 a 6 años. La tasa de concepción de las alpacas del Grupo B fueron mayores que las del Grupo A, aunque sin diferencia significativa (Cuadro 2). En relación al tiempo de cópula, en el promedio general se observó una mayor tasa de concepción conforme aumentaba el tiempo de cópula, determinando diferencias significativas $(\mathrm{p}<0.05)$ en tasa de concepción entre el Grupo A (48.3\%) y B (90.0) en los animales que emplearon más de 20 minutos en la cópula (Cuadro 2).

\section{Discusión}

Los resultados indican que si bien existe una mayor tasa de concepción en las alpacas con el mayor nivel sérico de estradiol en el momento de la monta, estas diferencias no fueron estadísticamente significativas y, por lo tanto, no puede señalarse que existe un efecto de los niveles de estradiol sobre la tasa de concepción; salvo en aquellos casos con tiempo de cópula superiores a los $20 \mathrm{mi}-$ nutos. Estos resultados se asemejan a los valores reportados por Deen et al. (2007) y por Young-Hai (1995) en camellos, quienes no encontraron elevaciones de estradiol en el momento del servicio y donde los niveles fueron muy bajos, basales e indetectables, a pesar de que los camellos presentaron folículos maduros.

En otras especies de rumiantes, la elevación de los niveles de estradiol durante la fase final de ciclo estral, además de ser responsables de la conducta de receptividad, tienen un importante rol en el proceso de estimular la secreción preovulatoria de la LH, y la consiguiente ovulación y formación del cuerpo lúteo (Noseir, 2003). Sin embargo, los resultados en alpacas indican que las tasas de concepción no estarían afectadas por el nivel sérico de estradiol inmediatamente después de la monta en alpacas, a pesar de presentar una conducta de receptividad sexual al macho. Es posible que el estradiol actúe sobre el cuerpo lúteo (CL) evitando su regresión como ocurre en los dromedarios, parientes cercanos de llamas y alpacas (Skidmore et al., 1997); sin embargo, la hormona de mayor importancia es la progesterona, necesaria para el mantenimiento de la gestación, ya que los camélidos sudamericanos son considerados CL dependientes (Sumar, 1988).

Los niveles promedio de estradiol $(0.78$ $\pm 0.39 \mathrm{pg} / \mathrm{mL}$ ) registrados en el presente estudio son bastante inferiores a los resultados reportados por Aba et al. (1995) en llamas $(15 \mathrm{pg} / \mathrm{ml})$; diferencia que puede ser atribui- 
da a diferencias en la técnica para la determinación hormonal; sin embargo, estos valores muestran cierta similitud con otros reportes en camellos (Deen et al., 2007).

Los resultados permiten sustentar la hipótesis que, en las alpacas, el estradiol parece desempeñar un rol importante para la conducta de receptividad al macho, pero no tiene efecto posterior sobre la secreción de LH, responsable de inducir la ovulación en las especies domésticas de ovulación espontánea y, por ende, no tendría efecto sobre la posterior formación del cuerpo lúteo y sobre la tasa de concepción, diferenciándose de otros rumiantes domésticos.

Aun cuando los mecanismos relacionados con la ovulación en camélidos no están claramente establecidos, estudios realizados señalan que la ovulación es inducida por la presencia de un Factor Inductor de Ovulación (FIO), presente en el plasma seminal (Adams et al., 2005). La presencia del FIO parece sustentar la hipótesis que el estradiol no sería una hormona clave en el desencadenamiento de la ovulación y la posterior formación y desarrollo del CL.

Aun cuando la monta por sí misma no induce un incremento en la secreción preovulatoria de estradiol, se ha reportado que la cópula estimula la producción del pico preovulatorio de LH para inducir la ovulación, pero probablemente inducido por el FIO presente en el eyaculado. En ovejas, el estradiol contribuye al pico de $\mathrm{LH}$, pero que no necesariamente se presenta un surgimiento como tal (Moenter et al., 1990).

Los niveles elevados de estradiol producidos por el folículo pre-ovulatorio inducen el comportamiento de celo; sin embargo, en algunas especies como ovejas y vacas, se requiere de la acción sinérgica con cantidades basales de progestágenos (Adams et al., 2008). Por otro lado, Arainga (2002) encuentra la presencia de folículos estrogénicos grandes $(>7 \mathrm{~mm})$ en alpacas con supervivencia embrionaria alrededor del día 12 post-ovula- ción. Esto indicaría que el efecto del estradiol es prácticamente nulo sobre la tasa de concepción en alpacas inmediatamente después de la cópula y antes de la ovulación, tal como lo demuestran los resultados.

\section{Conclusiones}

La tasa de concepción en alpacas con bajo nivel de estradiol en el momento de la monta $(<0.5 \mathrm{pg} / \mathrm{mL})$ fue de $52.5 \%$ y en alpacas con alto nivel de estradiol $(>0.5 \mathrm{pg} /$ $\mathrm{mL}$ ) fue de $66.7 \%$, sin diferencias estadísticas, indicando la ausencia de un efecto de los niveles de estradiol sobre la tasa de concepción.

\section{Literatura Citada}

1. Aba M, Forsberg M, Kindahl H, Sumar J, Edqvist L. 1995. Endocrine changes after mating in pregnant and non-pregnant llamas and alpacas. Acta Vet Scand 36: 489-498.

2. Adams G, Jaiswal R, Singh J, Malhi P. 2008. Progress in understanding ovarian follicular dynamics in cattle. Theriogenology 69: 72-80.

3. Adams GP, Ratto M, Huanca W, Singh J. 2005. Ovulation-inducing factor in the seminal plasma of alpacas and llamas. Biol Reprod 73: 452-457.

4. Araínga M, Leyva V, García W., Franco E. 2002. Efecto de la GnRH en el proceso de reconocimiento maternal de la preñez sobre la supervivencia embrionaria en alpacas. Rev Inv Vet, Perú 14: 104-110.

5. Bravo PW, Fowler ME, Stabenfeldt GH, Lasley BL. 1990. Ovarian follicular dynamics in the llama. Biol Reprod 43: 579-585.

6. Bravo PW, Stabenfeldt GH, Fowler ME, Lasley BL. 1992. Pituitary response to repeated copulation and/or Gonadotropin-releasing hormone administration in llamas and alpacas. Biol Reprod 47: 884-888. 
7. Deen A, Vyas S, Sahami MS, Saharan P, Sevta I, Chabra S. 2007. Estradiol17 ? and progesterone profiles of female camels at different reproductive stages. Israel J Vet Med. 62: 20-26.

8. Fernández Baca S, Hansel W, Novoa C. 1970. Embryonic mortality in the alpaca. Biol Reprod 3: 243-251.

9. Fernández Baca S. 1971. La alpaca: reproducción y crianza. Boletín de Divulgación $\mathrm{N}^{\circ} 7$. Lima: Centro de Investigación IVITA. 43 p.

10. Leyva V, García W. 1999. Efecto de la progesterona exógena sobre la función del cuerpo lúteo de alpacas. En: Res II Cong Mund Camélidos. Cusco. p 87.

11. Leyva V, García W. 2000. Efecto del estradiol (E2) en la fertilización y sobrevivencia embrionaria en alpacas. En: Res. XV Cong Nac Cienc Vet. Cusco. p 22.

12. Moenter SM, Caraty A, Karsch FJ. 1990. The estradiol-induced surge of gonadotropin-releasing hormone in the ewe. Endocrinology 127: 1375-1384.

13. Noseir WMB. 2003. Ovarian follicular activity and hormonal profile during estrous cycle in cows: the development of 2 versus 3 waves. Reprod Biol Endocrinol 1: 1-6.
14. Novoa C, Leyva V. 1996. Reproducción de alpacas y llamas. Publicación Científica $\mathrm{N}^{\circ} 26$. Lima: IVITA. 13 p.

15. Skidmore J, Allen W, Heap R. 1997. Maternal recognition of pregnancy in the dromedary camel. J Camel Pract Res. 4: 187-192.

16. Sumar J, Fredriksson G, Alarcón V, Kindahl H, Edqvist L. 1988. Levels of 15-keto-13, 14-dihydro-PGF ${ }_{2 \text { ? }}$, progesterone and oestradiol-17? after induced ovulations in llamas and alpacas. Acta Vet Scand 29: 339-346.

17. Sumar J. 1988. Removal of the ovaries or ablation of the corpus luteum and its effect on the maintenance of gestation in the alpaca and llama. Acta Vet Scand 83: 133-141.

18. Sumar J. 1997. Avances y perspectivas en reproducción de camélidos. En: Memorias I Symposium Internacional Avances en Reproducción de Rumiantes. Perú. p 30-55.

19. Young-Hai L. 1995. Progesterone and estradiol-17? proles in the peripheral blood plasma of bactrian camel during early pregnancy and pregnancy diagnosis. J Camel Pract Res 2: 53-54. 